No. 33, 31 de enero de 2022

ISSN impreso: 1390 - 6321

ISSN online: 2661 - 6734

\title{
Evaluación de Recursos Tecnológicos en la Banca Microempresarial de Machala para la concesión de créditos
}

\section{Evaluation of Technological Resources in the Microenterprise Bank of Machala for the granting of loans}

\author{
Grace Katiuska Viteri Guzmán ${ }^{1}$ \\ graceviterig@hotmail.com \\ ORCID: https://orcid.org/0000-0002-5645-2634 \\ Universidad Tecnológica Empresarial de Guayaquil \\ Claudia Margarita Avilés Ramírez ${ }^{2}$ \\ margaritaaviles81@hotmail.com \\ ORCID: https://orcid.org/0000-0002-9635-1088 \\ Universidad Tecnológica Empresarial de Guayaquil
}

Recibido: 28/9/2021, Aceptado: 28/12/2021

\begin{abstract}
RESUMEN
La presente investigación tiene como objetivo evaluar los recursos tecnológicos utilizados por las entidades bancarias de Machala en la concesión de créditos. La evaluación de dicha gestión se fundamenta desde dos perspectivas: la estratégica y la técnica, por ello se identifican dos modelos cuyas variables se relacionan entre sí en todos los aspectos, los cuales son: el modelo estratégico Balance Scorecard y la guía técnica para supervisión y control COBIT; como resultado de la aplicación de estos dos modelos, se consideró evaluar las cuatro metas establecidas por Cobit: la financiera, el cliente, los procesos internos y el aprendizaje, versus los tipos de tecnologías utilizados en la gestión de microcréditos. Para tal efecto se aplicó una metodología cuantitativa en la cual se analizó la información con la herramienta SPSS; los datos fueron recolectados de la población de diez entidades financieras de microcréditos en Machala. Los puntajes de evaluación obtenidos por cada grupo de tecnologías indican que el uso de las tecnologías claves lideran con respecto a las metas de Cobit que trata el aspecto de clientes y procesos internos, las tecnologías básicas son muy usadas en el aspecto financiero, y las tecnologías emergentes lideran en el aspecto financiero, procesos internos y de aprendizaje.
\end{abstract}

Palabras clave: microcrédito, recursos tecnológicos, modelos de Gestión de Tecnología, Balance SCORECARD, COBIT

\footnotetext{
${ }^{1}$ Máster en Educación Informática, Universidad de Guayaquil, Ecuador

2 Máster en Sistemas de Información Gerencial, UTEG, Ecuador
} 


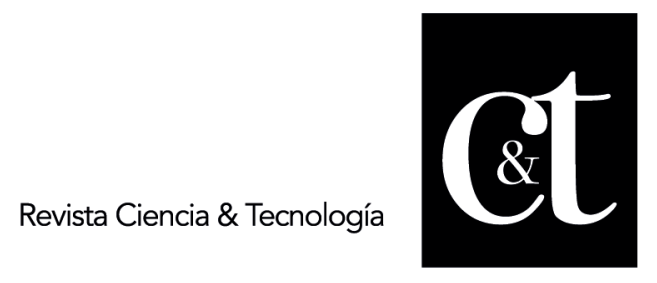

No. 33, 31 de enero de 2022

ISSN impreso: 1390 - 6321

ISSN online: 2661 - 6734

\begin{abstract}
The objective of this research is to evaluate the technological resources used by the banking entities of Machala in granting loans. The evaluation of this management is based on two perspectives: the strategic and the technical, for this reason two models are identified whose variables are related to each other in all aspects, which are: the Balance Scorecard strategic model and the technical guide for supervision and COBIT control; As a result of the application of these two models, it was considered to evaluate the four goals established by Cobit: financial, client, internal processes and learning, versus the types of technologies used in the management of microcredits. For this purpose, a quantitative methodology was applied in which the information was analyzed with the SPSS tool; the data were collected from the population of ten microcredit financial institutions in Machala. The evaluation scores obtained by each group of technologies indicate that the use of key technologies leads with respect to Cobit's goals that address the aspect of customers and internal processes, basic technologies are widely used in the financial aspect, and technologies Emerging companies lead in financial, internal and learning processes.
\end{abstract}

Keywords: microcredit, technological resources, Technology Management models, Balance Scorecard, COBIT

\title{
Introducción
}

En los últimos años, la economía del Ecuador ha sufrido situaciones bastantes complejas, desde los sucesos bancarios ocurridos a principios de 1999 hasta el desplome del precio del petróleo a $\$ 19,95$ en el 2016 (El Universo, 2016); esto obligó el cierre de empresas, cambios de otras y el nacimiento de muchas más. Hasta el año 2016, 843.745 empresas fueron registradas, y el 90,5\% del total corresponde a microempresas, aquellas con ventas menores a cien mil dólares por año, es decir un incremento de un 90\% en menos de 8 años. (INEC, 2017); siendo la actividad económica de mayor ponderación las actividades comerciales de mayoristas y minoristas.

El Oro ocupa el cuarto lugar en volumen de ventas a nivel nacional por detrás de Guayas, Azuay y Manabí. El origen de esto se debe a la producción bananera, mayor actividad de la provincia. Según el Ministerio de Agricultura, en la provincia de El Oro se concentra el $41,39 \%$ de las haciendas registradas en todo el país siendo la mayor parte propiedad de pequeños y medianos productores y generando 1.4 millones de toneladas de las de 6.2 toneladas registradas en todo el Ecuador. (Ministerio de Agricultura, 2017).

Como resultado de este crecimiento, el incremento de entidades financieras que faciliten el aumento de los microcréditos se ha convertido en un interés vital para las instituciones bancarias; quienes buscan captar clientes a través de las mejoras de servicios logradas gracias al avance de la tecnología. La adaptación de recursos tecnológicos a nivel empresarial ha pasado de ser un punto de vista técnico a convertirse en un punto de vista estratégico. 


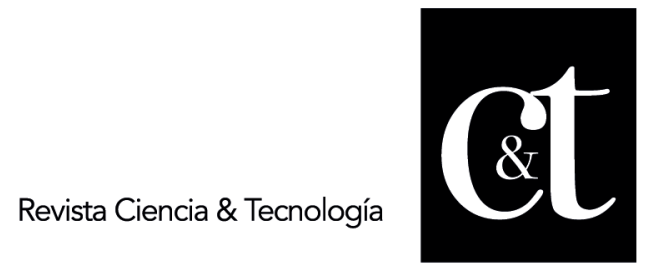

No. 33, 31 de enero de 2022

ISSN impreso: 1390 - 6321

ISSN online: 2661 - 6734

La aplicación de tecnologías de información no solo involucra herramientas, sino procesos tanto de adquisición y validación de recursos existentes y nuevos, ejecución de proyectos orientados a la mejora de servicios tanto para los usuarios externos como el soporte para los usuarios internos, con lo cual se crea un ambiente competitivo en el cual, el objetivo es lograr una ventaja traducida en crecimiento para la empresa y retorno de los valores invertidos (Torres et al., 2014).

\section{Microcrédito}

El término de Microcrédito no es algo novedoso, existe desde los años 60, naciendo en India y evolucionando continuamente. Cuando se habla de microcréditos, se pueden definir como aquellos préstamos cedidos a clientes de nivel social medio-bajo que presentan dificultades en conseguir un préstamo otorgado por una entidad bancaria. Los microcréditos surgen de una necesidad de impulsar el desarrollo de un país logrando que los microempresarios surjan como nuevas empresas cuyo referente son productos artesanales; su objetivo más importante combatir la pobreza y dar al microempresario un primer capital para iniciar su negocio (Escobar Pilataxi, 2015).

En el Ecuador, el microcrédito nace en la época de los años 70 a causa de desequilibrios en aspectos sociales y económicos; debido a esto, se inicia el cooperativismo, naciente en Guayaquil. Dado el crecimiento financiero el nacimiento de las Cooperativas de Ahorro y Crédito surgen y son registradas dentro de la Red Financiera Rural. Uno de los entes que han fomentado los microcréditos ha sido el Estado ecuatoriano, promoviendo la mayor parte de microcréditos en las ciudades más grandes del país y elevando esta actividad que se ve reflejada con un incremento importante de servicios de microcréditos, un ejemplo claro es el crecimiento de las instituciones financiera, cuyo número pasó de 14 entidades en el 2002 a 68 instituciones en el año 2010 (Escobar Pilataxi, 2015).

La asignación de un crédito debe ser otorgada en base a un estudio muy detallado del aplicante, aún si éste es considerado un microempresario, para esto, se sigue un proceso definido por las siguientes fases (Quezada Cueva, 2013):

Solicitud de Crédito: En esta primera fase, el cliente debe llenar los formularios de requerimiento de microcrédito, los mismos que incluyen información personal, de ambiente, tipos de ingresos y por su puesto información detallada del destino del dinero solicitado. Dentro de la información que el solicitante debe entregar se encuentra: La definición del monto, forma de pago, plazo, garantía para el crédito.

Análisis de Riesgo: Una vez realizada la solicitud, ésta, entra a una etapa de análisis, en la cual la entidad bancaria va a revisar y validar toda la información presentada, se solicitarán documentos adicionales si es necesario y se analiza el proyecto tanto como en su viabilidad determinando el nivel de riesgo de este. El proceso de análisis es el más largo y más detallado y se divide en tres subprocesos: 


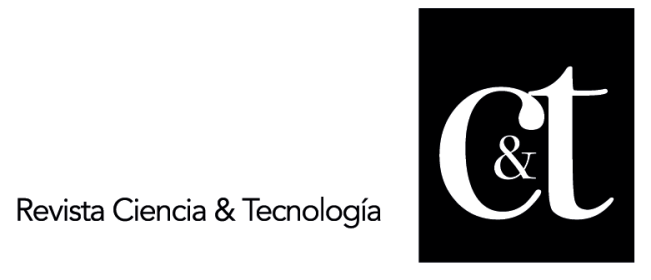

No. 33, 31 de enero de 2022

ISSN impreso: 1390 - 6321

ISSN online: 2661 - 6734

Verificación de la información: Consiste en la validación de información personal, tal como domicilio, teléfonos, garantías, descripción del negocio a iniciar, inventario.Análisis Cualitativo: incluye identificación de riesgos del negocio, identificar proveedores y clientes de este. o

Cuantitativo: Consiste en la verificación del monto, como se va a estructurar el plan de pagos de la deuda, evaluación del negocio, cuál será su volumen de ventas, los cosos asociados, margen de utilidad.

ión del Crédito: Una vez superada la fase anterior, pasa a un estado de validación final y aprobación, en donde se realiza un estudio final de toda la documentación presentada por el cliente: referencias bancarias, comerciales, verificación de información proveniente de la Central de Riesgos, y el asesor recomienda un monto, el cual, no necesariamente será igual al solicitado inicialmente.

$\bullet$

Adminis

tración y Desembolso: Se solicitan documentos adicionales previo al desembolso de dinero y se entrega el plan de pagos definido para el cliente en base a tiempos, montos e ingresos de este.

Los diferentes procesos generan mucha documentación y necesitan de la tecnología para cumplir todas las tareas especificadas. A continuación, en la tabla 1 un detalle promedio de los implementos utilizados en un proceso de crédito:

Tabla 1: Elementos participantes de un proceso de microcrédito

\begin{tabular}{|l|c|}
\hline \multicolumn{1}{|c|}{ Ítem } & Cantidad \\
\hline Documentos originales generados & 42 \\
\hline Copias generadas & 11 \\
\hline $\begin{array}{l}\text { Total de documentación general } \\
\text { (incluye formularios, información } \\
\text { personal del cliente) }\end{array}$ & 53 \\
\hline Personas participantes del proceso del & 10 \\
\hline $\begin{array}{l}\text { Entidades externas participantes del } \\
\text { proceso }\end{array}$ & 10 \\
\hline
\end{tabular}

Fuente: Quezada Cueva (2013)

\section{Recursos Tecnológicos}

Los recursos tecnológicos desde el punto de vista empresarial se definen como un paso hacia la madurez de una empresa; son herramientas que le permiten una renovación continua que permite incrementar las ventajas competitivas de sus negocios y a su vez lograr una adaptación total dentro de su entorno variable (Morin \& Seurat, 1999).

Para complementar dicho concepto, también podemos definir a los recursos tecnológicos como materiales tangibles, tales como equipos, herramientas, 


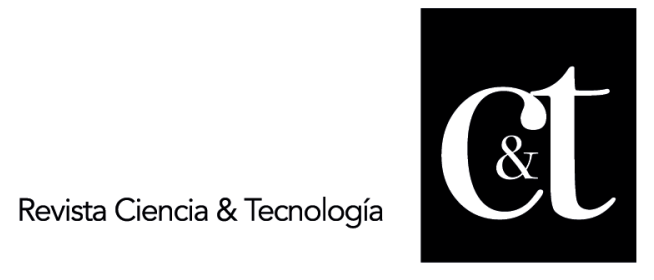

No. 33, 31 de enero de 2022

ISSN impreso: 1390 - 6321

ISSN online: 2661 - 6734

patentes, o materiales intangibles tales como técnicas, procedimientos científicos, conocimiento; propiedad de una empresa utilizable por un individuo, grupo de personas o sociedades con el fin de crear, comercializar y vender un producto (Mantulak et al., 2016).

Según los criterios de selección de tecnologías en base a la competitividad definidos por Arthur D. Little, las tecnologías desde el punto de vista estratégico y de proyectos se clasifican en los ítems detallados en la tabla 2 (Zartha et al., 2015).

Tabla 2: Clasificación de la Tecnología en base a la competitividad

\begin{tabular}{|l|l|}
\hline \multicolumn{2}{|c|}{ Desde el Punto de Vista Estratégico } \\
\hline Tecnologías & $\begin{array}{l}\text { Aquellas de quienes la compañía tiene } \\
\text { completo dominio, las conoce, las utiliza y } \\
\text { toma ventaja de todas sus funcionalidades, } \\
\text { gracias a ellas, la empresa tiene un nivel } \\
\text { de preponderancia en el mercado frente a } \\
\text { sus competidores. }\end{array}$ \\
\hline Tecnologías & $\begin{array}{l}\text { Aquellas que son necesarias para } \\
\text { desarrollar otras tecnologías o construir el } \\
\text { producto. Forman parte del ciclo de vida } \\
\text { del producto, aunque no generen una } \\
\text { ventaja competitiva en el mercado. }\end{array}$ \\
\hline Tecnologías & $\begin{array}{l}\text { Aquellas tecnologías nuevas, relacionadas } \\
\text { a la innovación total o parcial, de las cuales } \\
\text { se tiene poco dominio, por ello son } \\
\text { llamadas inmaduras presentando por ende } \\
\text { un nivel de riesgo mayor a las otras. }\end{array}$ \\
\hline
\end{tabular}

Fuente: Zartha et al. (2015)

\section{Modelos de Gestión de Tecnología}

La gestión de Tecnología consiste en un sistema de administración de conocimiento compuesto por dos partes: el dominio de la práctica y la aplicación de procesos que incluyan planificación, organización ejecución y control. Todo proyecto de Tecnología debe ser ejecutado bajo un proceso de gestión; este proceso debe asegurar la selección, control y evaluación de los recursos. El proceso de Gestión de Tecnología se basa en estos tres aspectos, los cuales fueron definidos por la Organización de Cooperación y Desarrollo Económicos de Estados Unidos (Naciones Unidas, 2005).

Selección: Consiste en buscar, analizar y medir riesgos, rentabilidades y la manera en la que el proyecto va a contribuir positivamente a la empresa.

Control: Consiste en el monitoreo continuo de costos, tiempos y productividad de los recursos.

Evaluación: Revisión de lo implementado y realizar correcciones si son necesarias.

Al hablar de gestión de recursos tecnológicos a nivel empresarial, ésta se divide en dos partes: Gestión Estratégica de Tecnología y Gestión Operativa de Tecnología. 


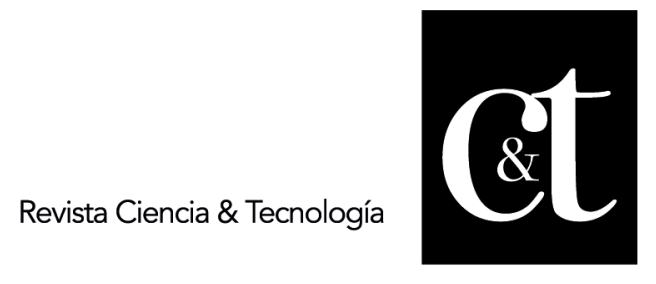

No. 33, 31 de enero de 2022

ISSN impreso: 1390 - 6321

ISSN online: 2661 - 6734

\section{Gestión Estratégica de Tecnología}

Consiste en la alineación de las políticas y objetivos estratégicos de la empresa y los recursos tecnológicos a fin de lograr el cumplimiento de las metas establecidas y la mejora de los productos. La gestión estratégica de tecnología ha evolucionado hasta convertirse en uno de los puntos más importantes dentro de un proceso de negocio. La gestión estratégica de Tecnología se basa en las siguientes políticas (Rincón Bermúez \& Peláez Ramírez, 2013):

La definición de objetivos estratégicos de la empresa debe definirse antes de cualquier compra de tecnología.

La adquisición de la tecnología se basa en un proceso debidamente establecido cuya meta principal sea la cobertura de todas las necesidades del negocio.

Definir los objetivos de la gestión de la tecnología, tomando en consideración la estructura de la empresa.

Identificar talentos relacionados a la tecnología entre los recursos humanos.

Definir los diferentes actores a lo largo del proceso de adquisición de tecnología.

Evaluar nueva tecnología que pueda aportar positivamente a los objetivos estratégicos.

Planificar adquisiciones o implementación de tecnología sea esta nueva o existente.

\section{Modelo de Gestión Tecnológica basado en Balance Scorecard}

El Balance Scorecard es modelo para analizar y definir estrategias organizacionales propuesto por Kaplan y Norton en 1992; está compuesto por cuatro ítems generales o perspectivas como lo muestra la figura 1 , de las cuales tres no se orientan al ámbito financiero y solo una sí. El punto de vista financiero es quien determina los indicadores económicos que validan el modelo y quienes pueden ayudar a la toma de decisiones que involucren iniciativas o incrementos en el valor del negocio (Bento \& Bento, 2013) 


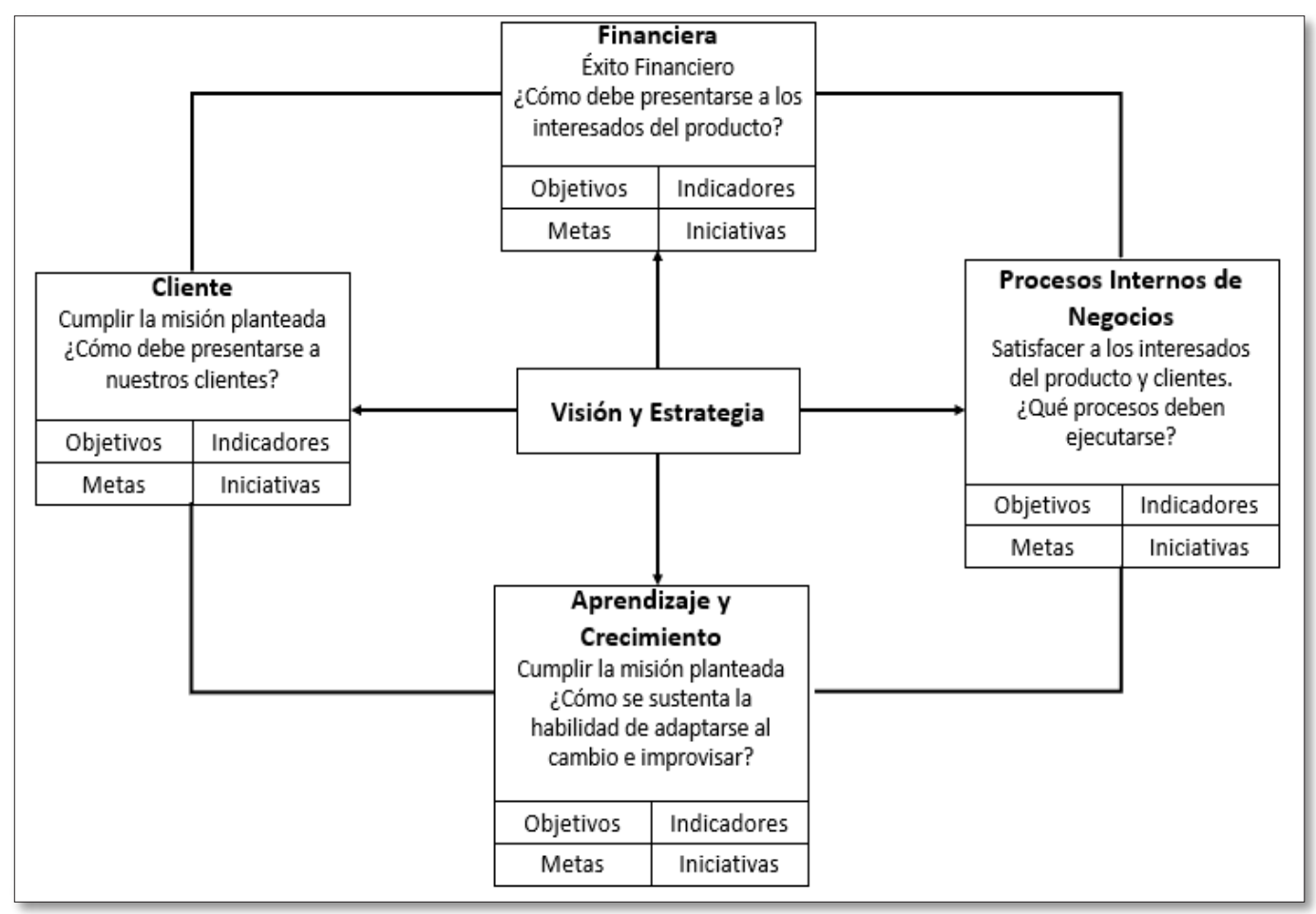

Figura 1: Modelo Balance Scorecard Kaplan-Norton Fuente: El Cuadro de Mando Integral (2009)

La perspectiva financiera se enfoca en el monitoreo de los incrementos económicos y la reducción de costos, es decir, el objetivo es maximizar el rendimiento financiero; la perspectiva de clientes se centra en el comportamiento del mercado, lograr la fidelidad del cliente logrando su plena satisfacción (Bento \& Bento, 2013).

En cuanto a la perspectiva de procesos, su orientación es interna; analiza e identifica la forma en cómo aplicar de la manera más eficaz ciertos procesos con el objetivo de incrementar la productividad; por último, la perspectiva de aprendizaje y crecimiento busca mejorar las habilidades técnicas del equipo humano de trabajo, es importante considerar que estas habilidades se encuentren alineadas a las metas estratégicas (Kalender Tugce \& Vayvay, 2016).

Este modelo de gestión se centra en lograr la alineación de los recursos tecnológicos y la gestión empresarial, con lo cual se logra obtener la mayor ventaja del uso de estos recursos con el fin de alcanzar las metas estratégicas propuestas. El análisis, la aplicación del modelo y las perspectivas correspondientes al Balance Scorecard: Financiera, Clientes, Procesos, Aprendizaje (Hernández Marcano, 2014).

\section{Gestión Operativa de Tecnología}

La gestión operativa, se basa en la adquisición de recursos. La decisión de la compra de tecnología se basa en la investigación y desarrollo quien a su vez 


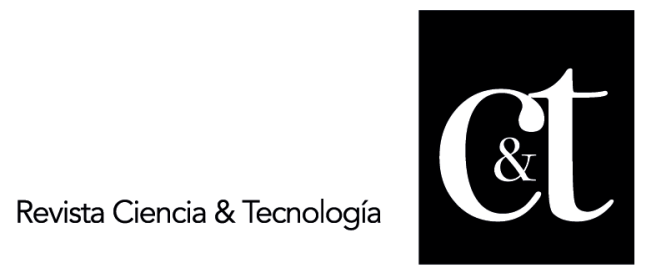

No. 33, 31 de enero de 2022

ISSN impreso: 1390 - 6321

ISSN online: 2661 - 6734

depende del movimiento del mercado y de los avances tecnológicos que existan.

\section{Modelo Cobit}

El modelo Cobit fue introducido en 1995 por ISACA, una organización encargada del fomento del conocimiento mediante educación y certificaciones, con el propósito de crear productos con un mayor promedio de vida en la visión negociadora de las empresas. La quinta versión publicada del proceso data del 2012 y se ha convertido en una herramienta muy popular a nivel mundial con clientes en más de 160 países. Cobit presenta 4 objetivos de alto nivel direccionados al control, los cuales son llamados los dominios para los procesos de tecnología: (Iza Verdesoto \& Vera Ayala, 2015)

Planeamiento y Organización. - Este dominio abarca estrategias, operaciones y tácticas relacionadas al entendimiento del modo en el cual la tecnología se relaciona con la visión estratégica.

Adquisición e Implementación. - Este dominio incluye las estrategias relacionadas a la determinación de cual tecnología adquirir o cual desarrollar considerando la facilidad de su aplicación a los procesos de negocio.

Entrega y Soporte. - Este domino se centra en el servicio operativo dedicado al soporte, mantenimiento de sistemas, procesamiento, almacenamiento de información.

Monitoreo y Evaluación. - Dominio que busca la validación de los otros dominios con el objetivo de asegurar la calidad a lo largo de la ejecución de cada uno de ellos.

Cobit 5 corresponde a la quinta versión de este modelo y se basa en cinco principios (ISACA, 2017):

Satisfacer las necesidades de los interesados del negocio: Consiste en proporcionar herramientas para generar y conservar el valor del negocio alineando las metas TI con las metas corporativas.

Abarcar todas las secciones de la empresa: Mediante sus procesos, actividades y tareas Cobit considera todas las funciones de la empresa incluyendo todos los departamentos y personal humano participante.

Establecer un marco de trabajo integrado: Dado el principio anterior permite personalizar los procesos, aplicar estándares en función de las metas operacionales. 


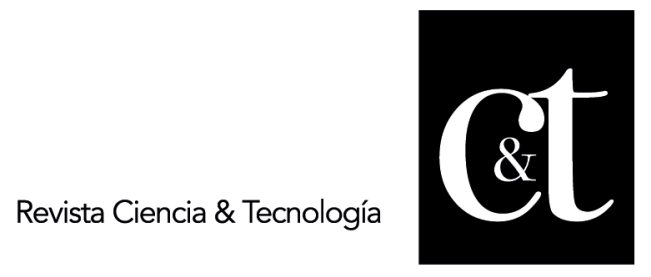

No. 33, 31 de enero de 2022

ISSN impreso: 1390 - 6321

ISSN online: 2661 - 6734

Permitir un enfoque holístico: Consiste en la inclusión de secciones interactivas que dan soporte al sistema de Gestión de TI.

Identificar diferencias entre el gobierno y la gestión: Acorde a Cobit, el gobierno se relaciona al interesado del producto, sus necesidades, especificaciones, y la gestión se centra en la administración de todos los recursos de forma efectiva para cumplir los requerimientos establecidos.

El Modelo Cobit 5 trabaja bajo un esquema de metas establecidas de modo que las estrategias organizacionales y las metas IT se relacionen entre sí. La lista de las metas se detalla en la tabla 3.

Tabla 3: Metas Cobit relacionadas con las TI

\begin{tabular}{|c|c|c|}
\hline $\begin{array}{l}\text { Dimensión del } \\
\text { CMI TI }\end{array}$ & \multicolumn{2}{|c|}{$\begin{array}{c}\text { Meta de Información y Tecnología } \\
\text { Relacionada }\end{array}$} \\
\hline \multirow{6}{*}{ Financiera } & 01 & $\begin{array}{l}\text { Alineamiento de TI y } \\
\text { Estrategia de negocio. }\end{array}$ \\
\hline & 02 & $\begin{array}{l}\text { Cumplimiento y Soporte } \\
\text { de la TI al cumplimiento } \\
\text { del negocio de las leyes } \\
\text { y regulaciones externas. }\end{array}$ \\
\hline & 03 & $\begin{array}{l}\text { Compromiso de la } \\
\text { dirección ejecutiva para } \\
\text { tomar decisiones } \\
\text { relacionadas con TI. }\end{array}$ \\
\hline & 04 & $\begin{array}{l}\text { Riesgos de negocio } \\
\text { relacionados con las TI } \\
\text { gestionados. }\end{array}$ \\
\hline & 05 & $\begin{array}{l}\text { Realización de } \\
\text { Beneficios del portafolio } \\
\text { de Inversiones y } \\
\text { Servicios relacionados } \\
\text { con las TI. }\end{array}$ \\
\hline & 06 & $\begin{array}{l}\text { Transparencia de los } \\
\text { costes, beneficios y } \\
\text { riesgos de las TI. }\end{array}$ \\
\hline & 07 & $\begin{array}{l}\text { Entrega de servicios de } \\
\text { TI de acuerdo con los } \\
\text { requisitos del negocio. }\end{array}$ \\
\hline Cliente & 08 & $\begin{array}{l}\text { Uso adecuado de } \\
\text { aplicaciones, } \\
\text { información } \\
\text { soluciones tecnológicas. }\end{array}$ \\
\hline & 09 & Agilidad de las TI. \\
\hline Interna & 10 & $\begin{array}{lr}\text { Seguridad de la } \\
\text { Información, } \\
\text { infraestructura } \\
\text { procesamiento } \\
\text { aplicaciones. }\end{array}$ \\
\hline Hiteria & 11 & $\begin{array}{l}\text { Optimización de activos, } \\
\text { recursos y capacidades } \\
\text { de las TI. }\end{array}$ \\
\hline & 12 & $\begin{array}{l}\text { Capacitación y soporte } \\
\text { de procesos de negocio } \\
\text { integrando aplicaciones }\end{array}$ \\
\hline
\end{tabular}




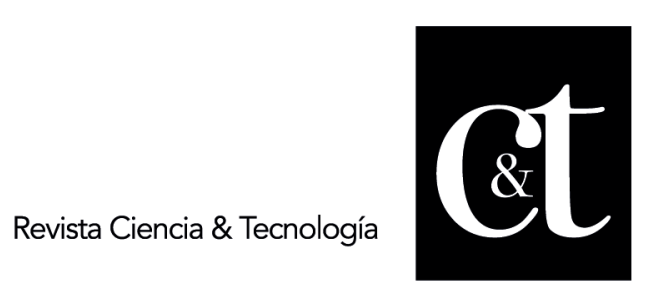

No. 33, 31 de enero de 2022

ISSN impreso: 1390 - 6321

ISSN online: 2661 - 6734

\begin{tabular}{|c|c|c|}
\hline & & $\begin{array}{l}\text { y tecnología en procesos } \\
\text { de negocio. }\end{array}$ \\
\hline & 13 & $\begin{array}{l}\text { Entrega de Programas } \\
\text { que proporcionen } \\
\text { beneficios a tiempo, } \\
\text { dentro del presupuesto } \\
\text { y satisfaciendo los } \\
\text { requisitos y normas de } \\
\text { calidad. }\end{array}$ \\
\hline & 14 & $\begin{array}{l}\text { Disponibilidad } \\
\text { información útil y fiable } \\
\text { para la toma de } \\
\text { decisiones. }\end{array}$ \\
\hline & 15 & $\begin{array}{l}\text { Cumplimiento de las } \\
\text { políticas internas por } \\
\text { parte de las TI. }\end{array}$ \\
\hline \multirow[b]{2}{*}{$\begin{array}{c}\text { Aprendizaje y } \\
\text { Crecimiento }\end{array}$} & 16 & $\begin{array}{l}\text { Personal del negocio y } \\
\text { de las TI competente y } \\
\text { motivado. }\end{array}$ \\
\hline & 17 & $\begin{array}{l}\text { Conocimiento, } \\
\text { experiencia e iniciativas } \\
\text { para la innovación de } \\
\text { negocio. }\end{array}$ \\
\hline
\end{tabular}

Fuente: ISACA (2017)

Uno de los cinco principios establecidos se centra en la diferencia entre Gobierno y Gestión; en base a estas diferencias, los procesos del Cobit 5, se definen en dos grupos (figura 2).

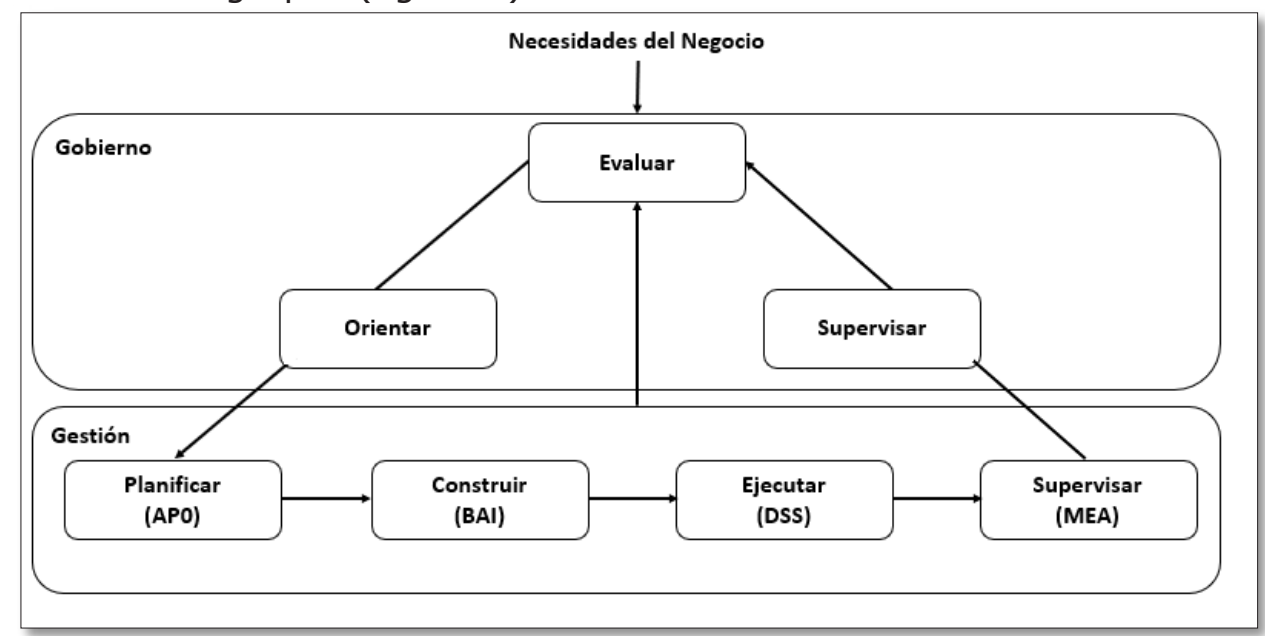

Figura 2: Áreas de Gobierno y Gestión de Cobit 5

Fuente: (ISACA, 2017)

Los procesos de Gobierno son tres, los cuales se incluyen reglamentos para orientar, supervisar y evaluar objetivos relacionados con las necesidades de los interesados; los procesos de gestión se componen de 4 grupos, de los cuales,

60

Viteri y Avilés. Evaluación de Recursos Tecnológicos en la Banca Microempresarial de Machala para la concesión de créditos 


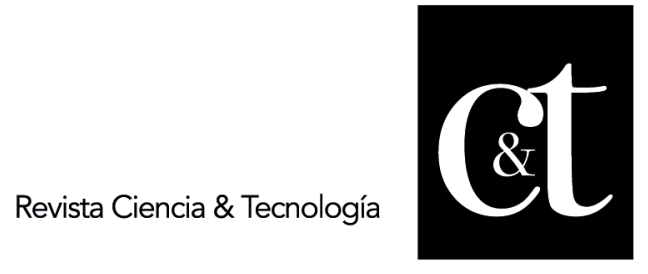

No. 33, 31 de enero de 2022

ISSN impreso: 1390 - 6321

ISSN online: 2661 - 6734

los procesos de planificación, construcción y ejecución se centran en gestionar tiempos, actividades y tareas que permitan cumplir dichas necesidades y los procesos de supervisión en vigilar que todas las actividades independientemente de la unidad de negocio o naturaleza cumplan lo estipulado en el estándar. (Bayas Condo \& Lozada Sánchez, 2014)

\section{Metodología}

Se aplicó un tipo de investigación descriptivo por cuanto se identifican variables relacionadas con los lineamientos estratégicos de los bancos; esto se logró a su vez con la aplicación del método deductivo considerando fuentes de entrada de datos provenientes de la observación y recolección de información de dichas instituciones. Se identificó el modelo apropiado para la evaluación de la gestión de los recursos tecnológicos y con los datos estadísticos recolectados se realizó un análisis cuantitativo para obtener los criterios más importantes a ser considerados para lograr una buena gestión que aporte crecimiento y mejoras a los procesos de microcréditos.

Para esta investigación se aplicó el método cuantitativo dado que se establecieron indicadores de medición de las variables bajo las cuales se realiza la evaluación de la gestión; estos provienen de datos estadísticos recolectados de las entidades bancarias analizadas en Machala medidos bajo las perspectivas: Financiera, Cliente, Procesos Internos y Aprendizaje (Sánchez, 2013). Se obtuvieron porcentajes de comportamiento por cada una de las perspectivas y a su vez porcentajes de cumplimiento de la gestión de recursos tecnológicos, así mismo se utilizó el método analítico para tomar estos porcentajes resultantes por cada indicador y subindicador e interpretarlos para lograr obtener los resultados de la evaluación.

Entre las técnicas cuantitativas utilizadas se encuentran:

Estadística. - Corresponde a métricas obtenidas de reportes resultantes de evaluaciones de recursos tecnológicos realizados en entidades bancarias de Machala.

Documental. - Corresponde a métricas que provienen de reportes de evaluación obtenidos de tesis relacionadas a la gestión de recursos tecnológicos realizados para universidades o centros de estudios

Se consideró como muestra la población total de 10 entidades bancarias que, según la Superintendencia de Bancos operan en Machala entregando servicios de microcréditos, por lo cual no es necesario obtener una proporción muestral. El número de entidades bancarias se definen a continuación en la tabla 4:

Tabla 4: Detalle de Muestra por Tipo de Entidad Bancaria

\begin{tabular}{|l|c|}
\hline $\begin{array}{l}\text { Tipo de Entidad } \\
\text { Bancaria }\end{array}$ & Cantidad \\
\hline Pública & 2 \\
\hline Privada & 8 \\
\hline
\end{tabular}

Fuente: Superintendencia de Bancos (2018) 


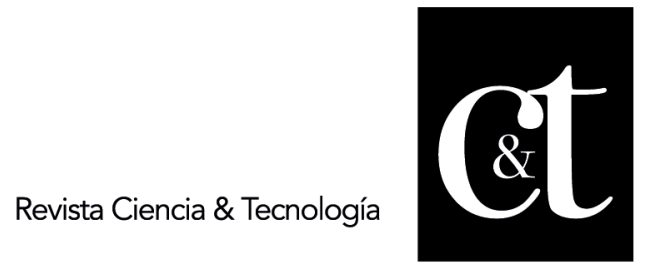

No. 33, 31 de enero de 2022

ISSN impreso: 1390 - 6321

ISSN online: 2661 - 6734

\section{Resultados y discusión}

Los recursos tecnológicos fueron divididos en tres grupos: claves, básicos y emergentes, y evaluados en dos perspectivas: la administrativa aplicando el modelo Scorecard y el técnico por medio del modelo Cobit 5 puesto que ambos presentan variables acoplables entre sí: planificación, calidad, personal y control, los cuales se encuentran alineados a la gestión de proyectos; aunque los factores de tiempo y alcance se encuentran también alineados no son considerados puesto que no son variables.

La evaluación con de las tecnologías claves presenta un mayor porcentaje de cumplimiento en la variable de procesos internos, dado que este tipo de tecnología engloba las herramientas de sistemas referentes software prioritario en procesos de evaluación y gestión de microcréditos, el peso más importante de su uso recae a lo largo de la ejecución de los procesos internos y permite que éstos se ejecuten con mayor eficiencia. Como segunda variable de importancia, tenemos la variable de cliente, indicando que las tecnologías claves permiten que los clientes perciban en un alto grado de positivismo la mejora obtenida de los procesos internos; en cuanto a la variable financiera, ésta se presenta en tercer lugar, reflejando la importancia de la inversión realizada, la cual ha obtenido frutos dado el porcentaje alto en procesos internos. Por último, se encuentra la variable de aprendizaje, el hecho de que esta variable se encuentre en un $43 \%$ presentando una brecha bastante amplia con respecto a las tres anteriores, refleja que la capacitación se realizó, pero no en un grado significativo; aun así, esto no afectó a los procesos internos.

La evaluación de las tecnologías básicas presenta un mayor porcentaje de cumplimiento en la variable de procesos internos, al igual que las tecnologías claves, las básicas son sumamente importantes para la ejecución de los procesos internos, como resultado de esto podemos apreciar que el porcentaje de cumplimiento de procesos internos es ligeramente superior a las tecnologías básicas. En segundo lugar, muestra un grado de importancia la variable financiera, puesto que para la alta gerencia el funcionamiento adecuado de estos tipos de tecnologías es importantes puesto que corresponden la base de las actividades bancarias; en tercer lugar, se presenta la variable de cliente, este tipo de tecnologías no interactúa mucho con los clientes y evita que ellos puedan percibir un beneficio directo de las mismas. Por último, la variable aprendizaje, en cuanto este indicador, no es tan importante como en la tecnología anterior, puesto que los usuarios internos están totalmente familiarizados con las herramientas de este grupo y por ende no es un requerimiento importante afianzar destrezas ya ganadas en este aspecto.

La evaluación de las tecnologías emergentes muestra una mayor preponderancia en la variable de aprendizaje con un $69.89 \%$, como resultado de las innovaciones aplicadas, la correcta capacitación de estas permite la mejora de los procesos internos, por ello es que esta variable presenta un mayor porcentaje de cumplimiento en la variable de procesos internos logrando que ésta se presente en segundo lugar con un $69.28 \%$. 


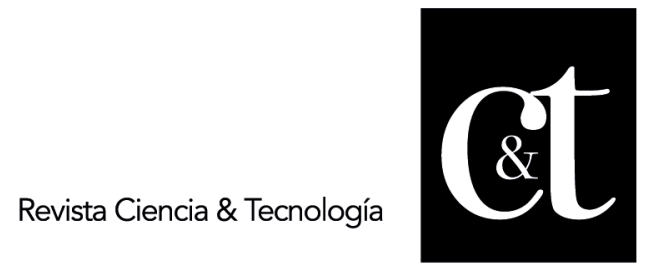

No. 33, 31 de enero de 2022

ISSN impreso: 1390 - 6321

ISSN online: 2661 - 6734

Si bien es cierto, estas tecnologías deberían tener una mayor influencia en los clientes finales, vemos que ésta ocupa el cuarto lugar con un $54 \%$, dejando a la variable financiera en un tercer lugar con un $57.29 \%$; puesto que este tipo de tecnología involucra una mayor inversión y riesgo para las operaciones financieras, este tipo de tecnología involucra un mayor seguimiento y evaluación por la directiva, puesto que los resultados deben ser monitoreados continuamente para poder establecer si una innovación es viable o no.

\section{Conclusiones}

Por todo lo expuesto en esta investigación se concluye que se debe identificar en primer lugar el tipo de recurso tecnológico y organizar por grado de importancia las variables de evaluación de dichos proyectos de microcrédito. Las variables de evaluación deben ser identificadas y medidas en base al objetivo para el cual ese recurso fue concebido como parte de la entidad financiera. Y por último considerar los criterios relevantes por tipo de tecnología a evaluar, así como sus variables; adicional es importante que la variable relacionada al cliente sea considerada preponderante para las tecnologías claves y emergentes puesto que éstas entran en relación directa con el usuario final, así como el usuario interno permitiendo que los procesos de crédito fluyan, se representen en mayores ingresos y menores tiempos de evaluación de dichos créditos.

\section{Referencias bibliográficas}

Bayas Condo, M. I., \& Lozada Sánchez, W. G. (2014). Desarrollo de un modelo de madurez tecnológico para categorizar a las instituciones financieras de los segmentos 3 y 4 de la Superintendencia Economía Popular Solidaria. Obtenido de http://repositorio.espe.edu.ec/bitstream/21000/9664/1/T-ESPE048258.pdf

Bento, A., \& Bento, R. (mayo de 2013). Validating Cause and Effect Relationships in the Balance Scorecard. Academy of Accounting and Financial Studies Journal, 13. Obtenido de www.researchgate.com: https://www.researchgate.net/publication/259460965_Validating_Causeand-Effect_Relationships_in_the_Balanced_Scorecard

El Universo. (2016). El precio internacional del crudo históricamente ha sido bajo. El Universo, $2 . \quad$ Obtenido de https://www.eluniverso.com/noticias/2016/01/24/nota/5362303/preciointernacional-crudo-historicamente-ha-sido-bajo

Escobar Pilataxi, J. W. (2015). Evaluación del Impacto del Microcrédito en la reducción de la pobreza en el Ecuador. (Tesis de grado, Universidad de Guayaquil).

Hernández Marcano, J. J. (abril de 2014). Desarrollo de un modelo de gestión tecnológico basado en el balanced scorecard para la integración de las tecnologías de información y comunicación (tic) a la praxis educativa de los docentes del Programa Nacional de Formación en Informática. Portal Educativo para las Américas. Obtenido de Portal Educativo para las Américas: http://recursos.portaleducoas.org/sites/default/files/VE14.082 $0 . p d f$ 


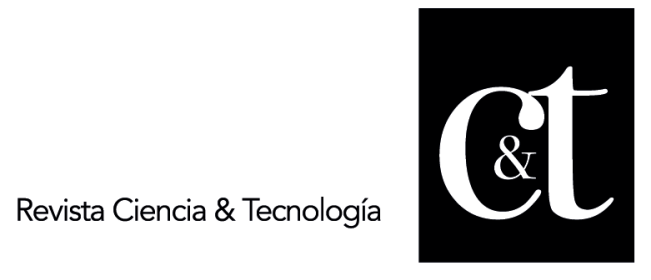

No. 33, 31 de enero de 2022

ISSN impreso: 1390 - 6321

ISSN online: 2661 - 6734

INEC. (2017). Ecuador en Cifras, 1. Recuperado el 31 de octubre de 2017, de http://www.ecuadorencifras.gob.ec/ecuador-registro-843-745-empresasen-2016/

ISACA (2017). Cobit 5 - Un Marco de Negocio para el Gobierno y la Gestión de las TI de la Empresa, Personalizado para la Ing. Margarita Avilés. ISACA.

Iza Verdesoto, M. d., \& Vera Ayala, M. E. (2015). Evaluación Técnica Informática de los Principales Procesos Utilizando los Dominios 3 y 4 de Cobit en el Banco Desarrollo $S$ A. (Tesis de maestría, ESPE). Obtenido de http://repositorio.espe.edu.ec/bitstream/21000/10818/1/T-ESPE049619.pdf

Kalender Tugce, Z., \& Vayvay, O. (2016). El Quinto Pilar del Balance Scorecard: Sustentavilidad. 12ava Conferencia de Administración Estratégica Internacional (pág. 8). Antalya: Universidad Marmara. Obtenido de https://ac.els-cdn.com/S1877042816315610/1-s2.0-

S1877042816315610-main.pdf?_tid=85b6ab17-feb4-485f-9c3eabaa692f8190\&acdnat $=1544152739 \_435$ e13f4c5b83b8ce2759136dbb31af 4

Mantulak, M. J., Hernández Pérez, G., \& Carlos, M. J. (diciembre de 2016). Gestión Estratégica de Recursos Tecnológicos en Pequeñas Empresas de Manufactura. Estudio de caso en Argentina. Visión de Futuro, 23. Obtenido de:

http://revistacientifica.fce.unam.edu.ar/index.php?option=com_content\&vi ew $=$ article\&id $=422 \&$ Itemid $=90$

Ministerio de Agricultura. (2017). http://sipa.agricultura.gob.ec/index.php/cifras-agroproductivas. Obtenido de http://sipa.agricultura.gob.ec: http://sipa.agricultura.gob.ec/index.php/cifras-agroproductivas

Morin, J., \& Seurat, R. (1999). Gestión de los Recursos Tecnológicos. COTEC.

Naciones Unidas. (2005). Manual de Organización Estadística. Obtenido de https://unstats.un.org/unsd/publication/SeriesF/SeriesF_28S.pdf

Quezada Cueva, K. J. (2013). Elaboración de un Plan Estratégico de Marketing para el Banco de Machala sucursal Loja. (Tesis de grado, Universidad Nacional de Loja). Obtenido de http://dspace.unl.edu.ec/jspui/bitstream/123456789/5949/1/Karen\%20Jac kelin\%20Quezada\%20Cueva.pdf

Rincón Bermúez, R., \& Peláez Ramírez, G. (diciembre de 2013). Adquisición de Tecnología: Un Modelo de Gestión. Revista Electrónica de Computación Informática, Biomédica y Electrónica, 18 . Obtenido de https://www.redalyc.org/html/5122/512251564001/index.html

Sánchez, J. C. (2013). Metodología de la Investigación Científica y Tecnológica. Díaz de Santos.

Superintendencia de Bancos, E. (2018). Ficha Crediticia Sectorial en Bancos Privados. Guayaquil: Superintendencia de Bancos. Obtenido de http://estadisticas.superbancos.gob.ec/portalestadistico/portalestudios/wpcontent/uploads/sites/4/downloads/2018/06/ES BP mar 2018.pdf 


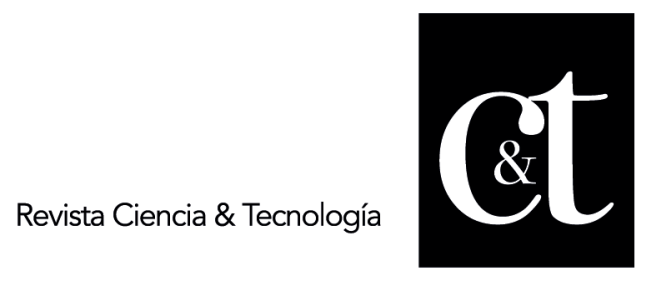

No. 33, 31 de enero de 2022

ISSN impreso: 1390 - 6321

ISSN online: 2661 - 6734

Torres Bermúdez, A. A., Arboleda, H., \& Lucumí Sánchez, W. (2014). Modelo de Gestión y Gobierno de Tecnologías de Información en Instituciones de Educación Superior. Grupo TIC Unicomfacauca, 2, 12.

Zartha Sosa, J. W., Álzate, A., Bibiana, H. Z., Henao, M., \& Gamaliel, J. (abril de 2015). Curvas en S y Análisis de Cluster en Ciclo de Vida de la Tecnología: Aplicación en 11 Tecnologías en Alimentos. Revista Espacios, 36, 5. Obtenido de http://www.revistaespacios.com/a15v36n12/15361206.html 


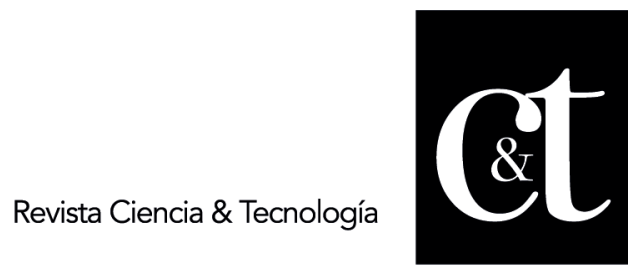

No. 33, 31 de enero de 2022

ISSN impreso: 1390 - 6321

ISSN online: 2661 - 6734

Viteri y Avilés. Evaluación de Recursos Tecnológicos en la Banca

Microempresarial de Machala para la concesión de créditos 